\title{
Mechanism of Social Reproduction of the Culture Futsal: Modelling of the Universals of Futsal and Sense of the Rules of the Game: Analysis of Shooting at the European Cup Matches
}

\author{
Nejah Kacem', Ayman Guemri' ${ }^{2}$, Chokri Naffeti ${ }^{3}$, Ali Elloumi1 \\ ${ }^{1}$ State, Culture and Change of Society, Higher Institute of Sport and Education Physics Ksar Saïd, \\ Mannouba, Tunisia \\ ${ }^{2}$ Group for the Study of Development and Social Environment (GEDES), Faculty of Human and Social \\ Science of Tunis, Tunis, Tunisia \\ ${ }^{3}$ Educational Department of PAS, Higher Institute of Sport and Education Physics of Sfax, Sfax, Tunisia \\ Email: kacemnejah@gmail.com
}

Received 23 February 2016; accepted 2 May 2016; published 6 May 2016

Copyright (C) 2016 by authors and Scientific Research Publishing Inc.

This work is licensed under the Creative Commons Attribution International License (CC BY).

http://creativecommons.org/licenses/by/4.0/

(c) (i) Open Access

\begin{abstract}
Genre and styles of Futsal playing: a comparison between the European and 2012 World Cups. This paper is the continuation of a research project started in 2012 to identify "genre" and "styles" of playing by national Futsal teams (Manno, 2012). Using a technological approach, the research addresses clinical activity concepts from an educational viewpoint that may be useful for teachers and coaches. With the assistance of a data collection method we compare various phases of the game during the 2012 World Cup with those observed in 2012. This comparison enables us to determine a "category" for each game. Furthermore, examination of how the teams' playing changed between CE and World Cups 2012 helps us to identify particular "styles". We then discuss our findings with a view of both initial and continuing training courses for sports teachers and Futsal coaches.
\end{abstract}

\section{Keywords}

Social Reproduction, Futsal, Modelling

How to cite this paper: Kacem, N., Guemri, A., Naffeti, C., \& Elloumi, A. (2016). Mechanism of Social Reproduction of the Culture Futsal: Modelling of the Universals of Futsal and Sense of the Rules of the Game: Analysis of Shooting at the European Cup Matches. Advances in Physical Education, 6, 59-66. http://dx.doi.org/10.4236/ape.2016.62007 


\section{Introduction}

Futsal is an amateur and professional sports, it is very developed (Doucet, 2012). It is a playful, physical, popular and educational activity. It has specific rules and is of real technical, tactical and mental training values of players. Although Futsal rules appear very clear, a professional experience is essential to determine the objective of internal game logic. We may be interested in Futsal through its own internal logic and its continuity for a methodological exploration game (case of analysis of the phase of finishing of the 2012 World Cup matches).

\section{Theoretical Approach}

Futsal and the playing technique: The almost constant contact with the ball and the speed of the game require a knowledge do and important skill. Control and manipulation of the ball most often occur with the sole for maximum grip on the ball. The control with the sole allows also continuing to visualize the placement and be located towards the area of teammate and opponent while keeping control of the ball. In this way it promotes the speed of the game (Derk et al., 2011).

The skill: The player must have acquired the necessary skill (shooting, control of the ball, coordination) to build and perform a pattern of activity that will produce the desired consequences. For example to succeed a shot on goal surrounded by opponents, control the ball in the race.

The perfect motivation: The player must have the optimum motivation needed to raise and maintain its investment until the purpose directing his learning activity is reached.

The motivation of choice: An important factor in success is the degree of personal investment towards the prescribed tasks. Self-confidence, the importance of the goal and anxiety are predictive of this investment. Motivation of choice takes place until the time or intend to learn is established. It therefore covers the process of goal setting, assessments of personal effectiveness and importance of the goal.

Executive motivation: Executive motivation is linked to the control of action implementation. It concerns the processes involved in maintaining an intention or purpose until it is accomplished and differs from the motivation of choices regarding, only the processes involved in the initial creation of an intent or purpose.

The environment: The player must have the cooperation of an environment that will facilitate or at least will not prevent progress towards the goal (Mohr et al., 2004). In other words, the environment (family, coach, condition of life,) must be in agreement with the personal goals of the player, capabilities cognitive, biological and transactional; it must provide the material resources and information necessary to facilitate the achievement of the goal and provide an emotional climate which supports and facilitates the efficient functioning.

Futsal and Athletic Performance (Adamson, 1962): Sports performance is the motor action whose rules are laid down by the sports institution allowing the subject to express its physical and mental potential.

Various factors interact more or less to achieve the performance (Bouthier, 1993). With this mind, we can consider that the coach is the only effective intervention for the athlete vector; allowing it to vary the planning, so to vary the training load according to the psychological and physical athlete.

The factors that influence athletic performance: Depending on the individual different learning abilities and are intimately related to the psychological and physiological factors.

The analysis of the requirement of the activity: Computerized matches observation facilitates detailed analysis of the skills needed in Futsal during competitions and training. It is therefore possible to obtain information on the movements and techniques or skills as well as their frequencies (contacts with the ball, passes, dribbles, tackles, jumps, turns etc...), distances and race times, intensities (high, low), the rythm of the stride, and the number of sprints, the game (with or without the ball), the durations of periods of work has low intensity (walking, jogging) the sense of moving forward, backward, side, etc.

Didactics of Futsal training: Education of skills techniques and strategies of Futsal can be a real challenge. All educators were confronted at a time gives to the question "what to say or do" with inexperienced players. Frustration can easily be done in the event of rapid failure. However, this is not always the inability of the player, but often has the method of the educator. With proper preparation, the educator will lead a meeting organized and designed to develop and increase learning. If a teacher enters the field with little direction, disruption arises and the results can frustrate the educator and the players. Educators have nearly 200 decisions to make in the session while teaching. The preparation and choice of themes appropriate will facilitate the holding of the session and learning.

Research question: Through the analysis of the Europe 2012 Futsal Cup matches can reveal a ranking in terms 
of efficiency of the different game than the results of matches?

Duration of the sequences, phases, actions: To quantify the number of sequence, phases. We are now concerned as to the duration of each sequence, phase, or action. Recall that a phase is the time during which the team has the ball. Each phase includes one or more actions. The sequences are suites of phases. They stopped when the ball is not in play.

Game sequences: A sequence is a succession of phase of attack-defence. A sequence starts when the ball is put into play and it stops when the ball is out of play. The alternation of phases of the game is sequences. This sequence stops when the ball is no longer in play. In the Futsal, the sequences do not include many phase. The game is quickly arrested.

Phases of the game: The number of actions by phase defines the style of play of the team and its approach to the opponent's goal. Can also be the average number of shares per phase for each team. You can also set the duration of each phase which is a criterion that defines the type of the conservation of the ball.

The duration of the phases: The phases include the linking of several successive praxies. A phase begins when the team recovers the ball and ends when it loses the ball. The duration of a phase is the time during which the team attacks. Medium phase durations measured in the observed matches the duration of phases varies with the style of play of the team. For example, the Russia team at the World Cup 2010 which the game was based on the collective conservation had phases that lasted on average 15 seconds. During Euro 2012 the Russia changed offensive animation and phases of the game were much faster. The duration of the phases varies with the style of game team (Doucet, 2012). The duration of a phase duration = action $\mathrm{x}$ number of actions. The actions of the top teams are much faster. This confirms that the Futsal players, instead of received idea, keep not individually the ball in the game. Do this to players who anticipate and just keep the ball. Consider instead the action as a praxie or pattern. Improve the conservation of the ball it is improve the technicality and gestural players meaning "know-how". The speed of the ball is one of the most important criteria in the tactical preparation of a team. To accelerate the ball it is necessary to have a set of features which are essential has the practice of Futsal: technical resources, tactical (or when, which, with whom) and physical resources (speed, strength). The objective of coach is that his team performs a perfect coordination between the use of the ball and the use of spaces. To do this, it will have to improve each player:

-(Motor and technical) know-how,

-The perception of the movement of the partners, adversaries,

-The speed of game which includes the speed of perception, reaction rate, the gestural frequency, the speed of execution

Modeling of a sports game: We present for illustrative purposes the main lessons learned from the institutional game modeling: volleyball. Its structure allows for two types of interaction: on the one hand, motor communication of solidarity manifested here in the form of passes between teammates and in another part, the motor against-communication of rivalry and which can to speak in one counter, lob, smash... Motor communications of volleyball universal can be summarised thus: between two players, there is never indifference: they are allies or opponents between two players, there is never of ambivalence: they cannot be at the same time partners and opponents between two players, during the entire game, the interaction is of a type unique, stable and always set by rule. Continuing its modelling work of high competition volleyball (Parlebas, 1988) was able to demonstrate, based on an exhaustive analysis of the 1986 World Championship matches, that some "evidence" of volleyball was not as obvious. Indeed, following a questionnaire sent to a sports population on the conduct of the games of volleyball, the results show that the percentage of errors in the answers is obviously high. The rigorous observation of the games was able to highlight the important role of service changes and was able to highlight a new parameter in the analysis of games: the number of crossings of net characterizing each game shots and whose influence depends on whether it is even or odd. By consulting studies of eminent experts on volleyball like (Parlebas, 1988), it realized that this author explains the fickleness of the duration of the games by the imperative of the two points of difference necessary to win the set and the obligation to win three sets. The analysis of the conduct of the games has shown that it is the change in service that is at the origin of this untimely duration of the games. Several researchers are interested in the study of football. According to their physiological, biomechanical and cultural axes, Futsal inspired from football which the specifics differ according to the particular rules of the discipline is not frequently used as research subject. Indeed, even if a read of the rules or the game may seem very clear, cannot stand today a personal experience and an experience in the Futsal to base findings or wear an objective eye on the current operation of the game. 
Hypothesis: Observation of the Futsal matches could reveal some substantive differences in relation to sociocultural designs of the game and a playful code which is not the same for all the world (Parlebas, 1998) "the playful contract is basically a social contract" can thus make the hypothesis that the Futsal practice has not been the same through time it is not a start with the practice of this sport from birth it is often inflected by the modification of the rules of game. We suggest that based on verifiable methods such as the rules of the game and motor behaviour on ground we can feel a game model for Futsal which determines the intelligibility of the game.

It is from the rules of the game that can enter the meaning of players' motor skills.

The analysis of matches (The coup of game): In our modelling approach, it gave considerable importance to the "coup of game" concept. The coup of game presents itself as the unit of basic analysis of sports games in this sense, share its delimitation between two settable terminals, it can fragment the game in many discontinuous segments and is involved in the establishment of universals and the creation of the model in the game. For the case of Futsal has defined the coup of game as motor sequence between two stops of game. More specifically, this sequence will extend from the moment when the ball becomes 'living' until the moment when the ball becomes "dead". The definition of the coup of game in terms of "closed" sequence essentially operates in a methodological relevance perspective. Thus when observed in a same time of game, several mounted of ball can occur without that there no goal scored, relevance lies in the fact of not to consider only the goal as a marker of analysis but rather the coup of game. Thus, it does not consider game kick as the action of a single team which completes his attack but rather motor interaction of the two teams which is in our opinion first, thus giving the praxic unit its intelligibility. However, there is very often Futsal, phases where the referee blows his whistle and the game "stops" to punish mistakes. The ball is considered to be alive. From there, one is led to consider the shot of 10 or 6 meters as a coup of game particular insofar as well its relevance in that it is a counter communication that occurs very often in the amendment that there is no actual interaction, the shot of 10 or 6 meters has relevance in the sense that it is a counter-communication that is very often involved in the modification of the score.

\section{Materials and Methods}

Our research is based on the observation of 8 matches from Futsal to a study population with the teams qualified to the final stage of a quarter of the final UEFA Futsal Championship. In sum, 16 Futsal teams subject to our analysis of the game to modelling for a total of about 224 players.

Procedures: The matches are cut to coup of game. It is proposed to analyze the coup of game in terms of duration, number of passes, from dribbling, steals, shooting, markdowns, value of coup of game as well as the percentage of the coup of game made, Sebastian (2012). More specifically, when viewing a Futsal match, it has available an observation grid. The first calculated parameter is the length of the coup of game. From a throw-in, for example, it raises the chronometer and expected there is stop for goal for whistled foul, for infringement for released ball, either game, or because the referee just stopped the game. There, we stop the chronometer and it counts the number of seconds. One brand on the grid the relative to the corresponding coup of game duration. Subsequently, we observed the other parameters, dribbling, passes, the markdowns, etc. and it between each stroke data from every coup of game, the coup of game being our analytical unit. For example, by number of passes per coup of game, there will be all the passes made by the two teams inside this coup of game. Here, it retains not only assists as that found usually in Futsal games statistics but rather all of the passes observed within the coup of game. By dribbling, means keeping the ball individually to advance on the ground or to overcome an opponent. The number of shots will include all the shots it is framed or not. The percentage of successful shots will be specified later during the preparation of the current style of game. The value of the coup of game means how many goal averages were marked by coup of game. In other words, how much is worth on average a game in terms of purpose? Percentage of successful coup of game, means all coup of game in which there were marked goals.

Measurement instruments: It will calculate the number of coup of game by match. Normally, by multiplying the number of the coup of game by their average duration, gets the approximate duration of the match (Beaufils, 1996). Thus, it has for our observation of the games of a grid of observation in the annex.

Materials: Playback of matches was done through CD. The observation of the matches was carried out on a TV.

Analysis, calculation and treatment operations are carried out through a portable microcomputer. The conduct 
of a game is not reduced to a catalogue of motor actions. It is also and above all a continuous sequence of motor behaviour, a possibility of passage from one to the other; it is a system of sub-roles. We can therefore establish the network of the movement of the motors socio sub-roles. This universal illustrates these sub-roles Combinatorics; it represents the dynamic multiple organization praxic paths offered to players, as it imposes the specific logic of the game. Any player of Futsal will necessarily circulate inside this network and borrow his own line within the common system. It is a fundamental invariant "revealing a limited number of sub-roles and any sports game lends itself to their networking in the form of universal" (Parlebas, 1998). The matrix of intelligibility of acts of game, it is the matrix of motors socio sub-roles. The kick is a complex technical element in implementation that required a lot of resources as much mental as physical and physiological. When shooting at the goal, more the ball going fast least goalkeeper has time to react. That's why the shoot must therefore give a highspeed ball so as to more easily deceive goalkeeper. The finishing: The finishing is materialized by a shot or a goal. It is the expression the game terminal and 1 logical outcome of actions. Shooting or goal succeeds to all the other technical elements of the game. It is to score a goal that the entire team works collectively and tries to place the "shooter" in the best conditions.

\section{Results}

From the analysis of Euro 2012 (qualification and finals), the 2012 World Cup and Euro 2014, we will try to determine important parameters of the finish and extract practical applications for training. Global statistics of observations confirm the intuitive observations. The teams who win mark more goals, are more efficient but not more often shoot at the goal. These averages vary competitions, but orders of size are the same.

The shots on goal are divided into 3 categories:

-The shots are finalizing by one goal,

-The unconverted frames shots,

-The shots not framed.

The average shoots observed during the final phase of the 2012 World Cup are divided depending on the results as shown in the Figure 1.

The teams that win have shoots 554 times the goal which $53 \%$ of fire executives while the teams that lose have shoots 424 times with $51 \%$ of fire executives. We can just conclude that teams who win more often shoot at the goal with the same proportion of framed shoots.

Shots not framed: The shots average non frames of the winning teams are higher (between 10 and 15) was one of the losing teams. The number of non framed shoot is not a significant parameter. It may be considered a loss of ball.

Framed shots: The difference between the averages of framed shots is significant so we are interested. Depending on the result the teams are divided according to 2 curves. Averages are in 10 to 15 shots framed for the teams who win and 15 to 20 fire frames for teams that lose. What tends to mean less need for framing of shots to win the winning teams players. It should be noted that a team has won within less than 5 shots. The majority of the winning teams are consistent between 10 to 15 shots per game.

Effectiveness of the framed shots: We can ask the question of whether the number of framed shots is correlated with the classification of Euro 2012 and what is the efficiency of the framed shots report for each team.

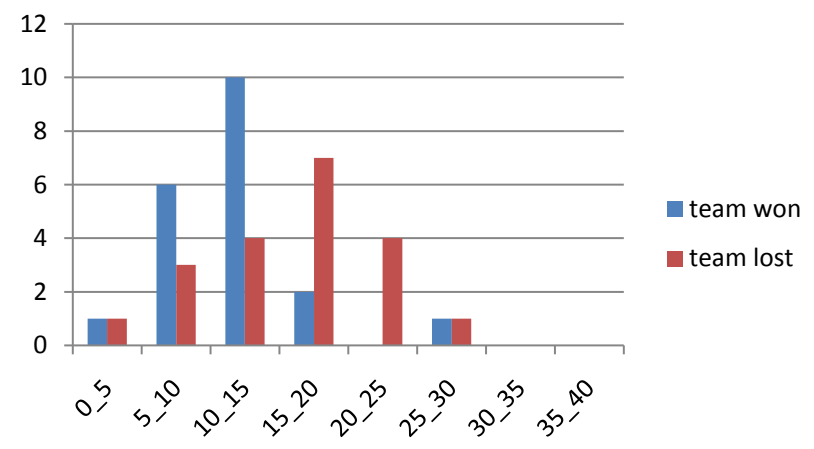

Figure 1. The average shoots observed during the final phase of the 2012 World Cup. 
The average efficiency rate is an average of $25 \%$ that a framed shot by 4 is converted to aim it takes at least 4 shots framed shot to mark.

Effectiveness of framed shots: Euro 2012 finals: 19.5\%. Is that the effectiveness rate is an important criterion?

Although the Ukraine team has the best efficiency rate, she has not qualified for the $1 / 4$ finals. However, teams of head correlate efficiency/number of scored goals brands more important than teams that lose. We therefore keep that these teams have high efficiency in the achievement of the kick. When the goals are scored: The distribution in time of the goals at the Championships of Europe of nations varies depending on competitions as shown in the following diagram. During Euro 2012, the number of goals marked in each 5-minute period varies little between $8 \%$ and $13 \%$ for every 5 minutes. On the basis of the halftime:

$-38 \%$ of the goals were marked in the first half;

$-62 \%$ in the second half;

$-20 \%$ of goals have marked in the last period from 40 to 45 minutes.

Distribution of goals on the basis of the result: It is more interesting to correlate the goals marks time for teams that win and, for those who lose. The distribution is proving different from the general distribution goals marked.

The teams who win tend to score at the beginning of each period that is within the first 10 minutes and between 20 and 30 minutes while teams who lose more mark late in the game. These results demonstrate that the concentration at the beginning of each half should be maximum and that the objective is to take advantage of the possible release of the opponent during the first 10 minutes of each half. As we have seen that fatigue has little impact at the end of match with high-level players. The concentration can be retained as an important criterion.

These observations can provide some information for training such as:

-Improve the effectiveness of the ability (improving the motor coordination, muscle elasticity.),

-Work the final actions from his own camp and not only shooting,

-To work the players by group Interline (pivot + wingers or fixed) with sequences a scope,

-Work the counter-attacks and the placement of the second player.

Identify youth as possible, those who have qualities of pivot that is:

-The meaning of the placement,

-The just moment,

-The execution speed.

\section{Discussion}

The major finding of this study was that the number of shots not framed was not a significant parameter. It may be considered a loss of ball. From the analysis of Euro 2012 (qualification and finals), the 2012 World Cup and Euro 2014, we will try to determine the important parameters of the finish and remove practical applications for training. Global statistics are based on observations that the winner team scored more goals. The winner shot 554 times on goal $53 \%$ of shots framed while the loser team fired only 424 times with $51 \%$ of framed shots. These averages vary in competitions, but orders of magnitude are the same.

We simply conclude that teams who win derive more often to the point with the same proportion of shots framed. In some competition the shooting efficiency factor varies $25 \%$ - 35\%. It should be noted that when the competition becomes eliminatory the effectiveness rate decreases. Is the effectiveness rate an important criterion?

In fact the efficiency by team report and the number of goals scored by team did not correlate as shown in the following diagram: Although the Ukraine team has the best efficiency rate, it has not qualified for the 1/4 finals. However, the top teams have a report effectiveness rate/number of most important goal marked only the teams that lose. We therefore observe that these teams have a high efficiency in the realization of the shot.

Depending on the result the teams divide following 2 curves of Gauss whose average crosses in 10 to 15 shots framed for winner team and 15 to 20 shots framed for teams that lose. That is mean the winner team needs less shots to win. They need a minimum of efficient of 5 shots. The majority of the winning teams are consistent between 10 to 15 shots per game. Starting from statistics, they define the probabilities of the result in depending on the number of goals scored by a team. Thus, if a team scores 4 goals it has $75 \%$ chance of winning and $5 \%$ chance of losing. To win, the team must return on the ground with the will of score at least 4 goals this is a difference with football or for the same probability the team must score 2 goals. The distribution in time of the 
goals in the European Championships of Nations varies depending on competitions as shown in the following diagram.

During Euro 2012, the number of goals scored in each period 5 minutes varies little 8\% and 13\% for every 5 minutes. The teams who win tend to mark at the beginning of each period i.e. the first 10 minutes and between 20 and 30 minutes, while teams who lose more mark at the end of match. In conclusion, these results demonstrate that the concentration at the beginning of each half should be maximum and that the objective is to take advantage of possible release of the opponent during the first 10 minutes of each half. As we have seen that fatigue has little impact at the end of match with high-level players. The low concentration is retained as an important criterion.

From a point of view, sport capacity, technical authority, addresses at the wheel are the basis of performance. The learning technique is a motor learning based on the regulating movements and cycle, feedback of actions and reactions. These are the psychomotor qualities that will be sought to acquire new techniques (Chevallard, 1991).

\section{References}

Adamson, M. (1962). Circuit Training. Ell and Sons Ltd., London.

Beaufils, B. (1996). Statistics Applied to Psychology. Volume 2: Inferential Statistics. Paris: Bréal. http://www.agro-montpellier.fr/sfds/CD/textes/zendrera1.pdf

Bouthier, D. (1993). Technology Access in S.T.A.P.S.: Performances and Teaching in Action APS. Diploma Habilitation Research. Orsay: Université Paris-Sud. https://www.researchgate.net/profile/Alain Mouchet/publication/269994028 Modlisation de la complexit des dcisions tactiques en rugby/links/549d710e0cf2d6581ab63c04.pdf

Chevallard, Y. (1991). The Didactic Transposition: Scientist Taught Knowledge (2nd ed.). http://rdm.penseesauvage.com/IMG/article_PDF/La-transposition-didactique.pdf

Derk, O. et al. (2011). Futsal Offers Accelerate Acquisition and the Development of the Technical Skills of Football and Some Mental Abilities Necessary to Succeed in Football in the Irish Players Aged Six to Eleven Years? http://www.fai.ie/domestic/news/o’neill-names-futsal-squad-for-uefa-qualifiers

Doucet et al. (2012) .The Bases of Futsal. Edulivre. http://www.aefoot.com/webroot/ef/les bases du futsal claude doucet.pdf

Mohr, M., Krustrup, P., Nybo, L., Nielsen, J. J., Bangsbo, J. et al. (2004). Muscle Temperature and Sprint Performance during Football Matches-Beneficial Re-Warm-up at Halftime. Scandinavian Journal of Medicine \& Science in Sports, 14, 156-162.

http://onlinelibrary.wiley.com/doi/10.1111/j.1600-0838.2004.00349.x/epdf?r3_referer=wol\&tracking_action=preview_cli ck\&show_checkout=1\&purchase_referrer=onlinelibrary.wiley.com\&purchase_site_license=LICENSE_DENIED

Parlebas, P. (1988). Analysis and Modeling of High Competition Volleyball: Analysis of the 1986 World Championships. Science and Motor. https://msh.revues.org/2968?file=1

Sebastian, M (2012). Planning Methodology, Principles, Feed. 
N. Kacem et al.

\section{Annexes}

Grid observation of the Futsal match:.

Event.............................

Result ....................................

\begin{tabular}{|c|c|c|c|c|c|c|c|c|c|c|c|c|}
\hline $\begin{array}{l}\text { Number } \\
\text { of blow } \\
\text { of the } \\
\text { game }\end{array}$ & $\begin{array}{l}\frac{\text { Length }}{\text { shot of }} \\
\frac{\text { the }}{\text { game }}\end{array}$ & Dribble & Pass & Interception & Unmarking & $\underline{\text { Shooting }}$ & $\frac{\text { Shooting }}{\text { Non }}$ & $\frac{\text { Penalty }}{\underline{10 \mathrm{~m}}}$ & $\frac{\text { Penalty }}{\underline{6 \mathrm{~m}}}$ & $\frac{\text { Cumulative }}{\underline{\text { fault }}}$ & $\frac{\text { Shot }}{\text { on }}$ & $\begin{array}{l}\begin{array}{l}\text { End } \\
\text { of the }\end{array} \\
\text { game } \\
\text { shot }\end{array}$ \\
\hline
\end{tabular}

G NG

1

2

3

4

5

6

7

8

9

10

11

12

13

14

15

16

17

18

19

20 